\title{
Augmented Rac1 Expression and Activity are Associated with Oxidative Stress and Decline of $\beta$ Cell Function in Obesity
}

\author{
Shutong Zhou ${ }^{\mathrm{a}, \mathrm{b}}$ Dongni Yu ${ }^{\mathrm{b}}$ Shangyong Ning ${ }^{\mathrm{c}}$ Heli Zhang $^{\mathrm{d}}$ Lei Jiang ${ }^{\mathrm{b}}$ Lei He \\ Miao Li ${ }^{b}$ Mingxiao Sun ${ }^{a, b}$ \\ aPeking University Fifth School of Clinical Medicine, Beijing Hospital, ${ }^{b}$ Department of Endocrinology \\ and Metabolism, Beijing Hospital; ' Cab of Hematology Department, Beijing Hospital; 'Department of \\ Endocrinology and Metabolism, Zhumadian Central Hospital; e Pathology Department, Beijing Hospital, \\ Beijing, China
}

\author{
Key Words \\ Rac1 $\bullet$ Oxidative stress $\bullet \beta$ cell dysfunction $\bullet$ Obesity
}

\begin{abstract}
Background: The aim of this study was to clarify the relationship among Rac1 expression and activation, oxidative stress and $\beta$ cell dysfunction in obesity. Methods: In vivo, serum levels of glucose, insulin, oxidative stress markers and Rac1 expression were compared between ob/ ob mice and $\mathrm{C} 57 \mathrm{BL} / 6 \mathrm{~J}$ controls. Then, these variables were rechecked after the administration of the specific Rac1 inhibitor-NSC23766 in ob/ob mice. In vitro, NIT-1 $\beta$ cells were cultured in a hyperglycemic and/or hyperlipidemic state with or without NSC23766, and the differences of Rac1 expression and translocation, NADPH oxidase(Nox) enzyme activity, reactive oxygen species (ROS) and insulin mRNA were observed. Results: ob/ob mice displayed abnormal glycometabolism, oxidative stress and excessive expression of Rac1 in the pancreas. NSC23766 injection inhibited the expression of Rac1 in the pancreas, along with amelioration of oxidative stress and glycometabolism in obese mice. Under hyperglycemic and/or hyperlipidemic conditions, Rac1 translocated to the cellular membrane, induced activation of the NADPH oxidase enzyme and oxidative stress, and simultaneously reduced the insulin mRNA expression in NIT-1 $\beta$ cells. Inhibiting Rac1 activity could alleviate oxidative stress and meliorate the decline of insulin mRNA in $\beta$ cells. Conclusions: Rac1 might contribute to oxidative stress systemically and locally in the pancreas in obesity. The excessive activation and expression of Rac1 in obesity were associated with $\beta$ cell dysfunction through ROS production.
\end{abstract}




\begin{tabular}{|c|c|c|}
\hline Cellular Physiology & Cell Physiol Biochem 2015;35:2135-2148 & \\
\hline and Biochemistry & $\begin{array}{l}\text { Dol: 10.1159/000374019 } \\
\text { Published online: April 07, } 2015\end{array}$ & $\begin{array}{l}\text { O } 2015 \mathrm{~S} \text {. Karger AG, Basel } \\
\text { www.karger.com/cpb }\end{array}$ \\
\hline
\end{tabular}

\section{Introduction}

The number of obese individuals has been increasing significantly worldwide in the past few decades [1]. Currently, obesity is considered a chronic disease that is characterized by a low level of inflammation-induced oxidative stress, which is regarded as the central component of the pathogenesis of obesity-related diseases, such as type 2 diabetes [2, 3]. NADPH oxidase (nicotinamide adenine dinucleotide phosphate-oxidase, Nox) is regarded as the major enzymatic source of oxidative products, as well as a multi-component protein that is closely implicated in the development of many obesity-related diseases [4, 5]. Recent evidence has indicated that Nox is highly activated in multiple diseases, including obesity and diabetes mellitus [6]. Rac1, a small guanosine triphosphate-binding protein that acts as an activator of Nox, has been implicated as playing a modulatory role in Nox-induced oxidative stress and mitochondrial dysfunction that is mostly caused by elevated glucose, lipids or cytokines $[7,8]$. In our previous study, we found that in monocytes, Nox was highly activated by enhancing Rac1 expression; obesity-induced oxidative stress and Rac1 expression were the consequence of aberrant glucose-lipid metabolism in overweight adolescents [9]. There was evidence that Rac1 contributed to vascular injury and cardiomyocyte apoptosis in the hyperglycemic state in people with diabetes [10], and Rac1 activation might lead to systemic and organic oxidative stress and apoptosis during hyperglycemia [11]. However, it is still unclear whether Rac1 acts in the pancreas, what the degree of relative oxidative stress is locally in the pancreas, and whether there is any further influence on $\beta$ cell function in obese individuals.

We hypothesize that oxidative stress exists not only systemically but also locally in the pancreas, which is primarily associated with the excessive expression and/or activation of Rac1. This may be one of the reasons for impaired $\beta$ cell function during the development of obesity, which might contribute to the progress of diabetes mellitus.

\section{Materials and Methods}

\section{Ethics Statement}

All animal studies were carried out in strict accordance with the recommendations in the Guide for the Care and Use of Laboratory Animals of the National Institutes of Health. The protocol was approved by the Animal Care and Use Committees of Peking University Health Science Center (Permit Number: IRB00001052-0711). All efforts were made to minimize suffering.

\section{In Vivo Studies}

C57BL/6J mice were obtained from the Laboratory Animal Center of Peking University. ob/ob mice were purchased from the Model Animal Research Center of Nanjing University. Mice were housed in microisolators under standard pathogen-free conditions on a $12 \mathrm{~h}$ light/dark cycle with free access to water and a standard chow. Mice were all male and 4 weeks old at the start of the experiment and were kept until 8 weeks old at the end of the experiment. Forty mice were divided equally into four groups: C57BL/6J as the normal control; ob/ob as the baseline for obesity; NSC ob/ob, which were ob/ob mice that were intraperitoneally injected with NSC23766 (2.5 mg. kg-1 . day-1, i.p.) for 2 weeks; and PBS ob/ob, which were ob/ob mice that received an equal volume of phosphate buffer solution (PBS) for 2 weeks.

\section{Biochemical Analysis}

Body weights were measured in all mice at 8 weeks of age. Blood glucose concentrations were tested after $12 \mathrm{~h}$ fasting from the tail veins of mice with a Roche blood glucose monitor (Glucotrend 2). Serum insulin was tested by chemiluminescence method (Immulite; DPC, Los Angeles, CA). Insulin resistance was estimated with the homeostasis model of insulin resistance $\left(\mathrm{HOMA}_{\mathrm{IR}}\right)$ by a formula that included fasting insulin $\left(\mathrm{I}_{0}\right)$ and fasting glucose $\left(\mathrm{G}_{0}\right)$ as follows: $\mathrm{HOMA}_{\mathrm{IR}}=\left(\mathrm{I}_{0} \times \mathrm{G}_{0}\right) / 22.5$ (insulin in $\mu \mathrm{U} / \mathrm{ml}$ and glucose in $\mathrm{mmol} / \mathrm{l}$ ). The levels of total cholesterol (TC) and triglyceride (TG) were measured using a kit from Roche (Basel, Switzerland). Malondialdehyde (MDA) was determined by the thiobarbituric acid method for the 


\section{Cellular Physiology Cell Physiol Biochem 2015;35:2135-2148 \begin{tabular}{l|l} 
and Biochemistry $10.1159 / 000374019$ & $\begin{array}{l}\text { Doblished online: April 07, 2015 } \\
\text { Puls Sarger AG, Basel } \\
\text { www.karger.com/cpb }\end{array}$ \\
\hline
\end{tabular} \\ Zhou et al.: Rac1 Associates with Oxidative Stress and $\beta$ Cell Dysfunction}

evaluation of systemic oxidative stress, and superoxide Dismutase (SOD) activity was calculated with a commercial assay kit (Cayman Chemicals, Ann Arbor, MI) as the marker of antioxidant ability.

\section{Immunohistochemistry Analysis of Pancreas}

C57BL/6J, ob/ob, NSC ob/ob and PBS ob/ob mice were anesthetized with isoflurane $(2.5 \%$ for 10 $\mathrm{min}$ ) at the age of eight weeks. Pancreases were carefully removed, fixed in formaldehyde and embedded in paraffin. Then, $4-\mu \mathrm{m}$-thick sections were cut in the horizontal plane. Serial sections were mounted onto glass slides, heat dried, deparaffinized in xylene, and dehydrated/rehydrated in a series of graded ethanols. The sections were stained with mouse anti-Rac1 primary antibody (Santa Cruz, 1:1000) overnight at $4^{\circ} \mathrm{C}$ in blocking solution, followed by fluorescein isothiocyanate conjugated anti-mouse IgG (Santa Cruz, 1:400) for $30 \mathrm{~min}$ at room temperature. All micrographs were taken under confocal microscopy.

\section{Western Blot Analysis of Pancreas}

Protein extracted from pancreatic tissue was prepared using a RIPA buffer. Protein separated by SDSPAGE was blotted onto polyvinylidene difluoride membranes. Blots were detected using Rac1 primary antibodies (Santa Cruz, 1:1000) and an Immobilon Western Chemiluminescent HRP substrate kit (Millipore). $\beta$-actin (Abcam, 1:4000) was used as the confidential reference item.

\section{Dihydroethidium (DHE) staining}

For DHE staining, the tissues were quickly embedded in Tissue-Tek ${ }^{\circledR}$ O.C.T. Compound (Sakura Finetechnical $\mathrm{Co})$ at $-20^{\circ} \mathrm{C}$. The O.C.T.-embedded tissues were sectioned $(10 \mu \mathrm{m})$ at $-20^{\circ} \mathrm{C}$ by a Benchtop Cryostat (Leica, CM 1100). After fixation, the slides were allowed to react with $5 \mu \mathrm{M}$ DHE at $37^{\circ} \mathrm{C}$ for 30 minutes. DHE-stained slides were mounted with VectaShield fluorescence mounting medium (Vector Laboratory). Images were captured with a Leica digital camera mounted on a fluorescent microscope attached to a PC running Leica 50TM software.

\section{In Vitro Studies and Measurement of ROS generation and NADPH oxidase enzyme activity}

NIT-1 $\beta$ cells were maintained at $37^{\circ} \mathrm{C}$ in a $\% \mathrm{CO} 2$ humidified incubator and incubated in Hanks' solution, pH 7.4. For various pharmacological treatments, glucose $(20 \mathrm{mM})$, palmitic acid $(100 \mu \mathrm{M})$, and both of the above were added into the cell suspension and kept for $6 \mathrm{~h}$, followed by $5 \mu \mathrm{M}$ dichlorodihydrofluorescein (DCF-DA) (Sigma) for $40 \mathrm{~min}$ at $37^{\circ} \mathrm{C}$. The control group was untreated. We washed NIT-1 $\beta$ cells three times with Hanks' solution and detected the fluorescence in cells by a flow cytometer. Then, on the basis of the aforementioned grouping, a highly selective Rac1 inhibitor, NSC23766 $(20 \mu \mathrm{M})$, was added to the three groups of cells overnight, and the fluorescence in NIT-1 $\beta$ cells was determined using flow cytometry.

The NADPH oxidase enzyme activity was measured using the method described by Hwang et al. [12]. Treated cells were homogenized using $50 \mathrm{mM}$ phosphate buffer solution ( $\mathrm{pH}$ 7.0) containing 1 mM EDTA and $1 \mathrm{mM}$ PMSF. The homogenates were centrifuged at $3000 \mathrm{~g}$ for $10 \mathrm{~min}$. The cleared lysates $(250 \mu \mathrm{g} / \mathrm{ml}$ of protein) were then incubated with N,N'-Dimethyl-9,9'-biacridinium dinitrate (Lucigenin) (5 $\mu \mathrm{M})$ for $2 \mathrm{~min}$ followed by the addition of NADPH $(100 \mu \mathrm{M})$. The chemiluminescence signal resulting from the reaction of superoxide anion and lucigenin was recorded every 1 min for 15 min using BioTek Synergy HT, Gen5. Nox enzyme activity was expressed as chemiluminescence units per mg lysate protein per minute.

\section{RNA extraction and reverse transcription}

The insulin mRNA from NIT- $1 \beta$ cells was extracted by Trizol reagent following the manufacturers instructions. Reverse transcription of RNA was performed with A3500 kit (Promega) according to the manufacturer's instructions.

\section{Immunofluorescence in vitro}

$1 \times 10^{5}$ NIT- $1 \beta$ cells were harvested and suspended with PBS, adhered to coverslips by a centrifugal slide stainer, fixed in pre-cold methanol at $-20^{\circ} \mathrm{C}$ for $10 \mathrm{~min}$, and then incubated with Rac1 primary antibodies (Santa Cruz, $1: 1000$ ) at $37^{\circ} \mathrm{C}$ for $60 \mathrm{~min}$. The cells were labeled with TRITC-conjugated anti-mouse IgG (Santa Cruz, $1: 400$ ) at $37^{\circ} \mathrm{C}$ for $60 \mathrm{~min}$ and incubated with DAPI at room temperature for 5 min. Finally, the coverslips were mounted with DABCO. Images were visualized with confocal microscopy.

\section{KARGER}




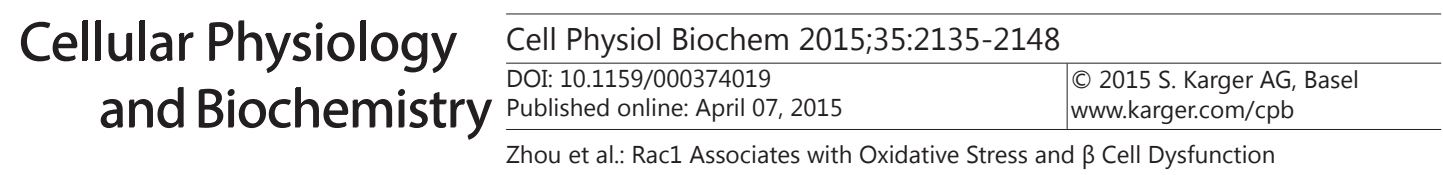

Statistical analysis

Data were expressed as mean values \pm standard deviations (SD). Comparisons between different groups were analyzed using a t-test for independent group comparisons. Statistical significance was set at $p$ $<0.05$. All data were analyzed with the SPSS 17.0 software.

\section{Results}

Obese mice displayed discrepancies in glycometabolism, lipid metabolism, and insulin sensitivity compared to normal weight mice

The body measurement, metabolic markers, and insulin sensitivity of the mice involved in this study are summarized in Table 1 . As expected, ob/ob mice displayed significantly higher levels of weight, Lee's index [13], fasting blood glucose (FBG), insulin, $\mathrm{HOMA}_{\mathrm{IR}}$, TG and TC compared with normal weight C57BL/6J $(p<0.05)$. These data indicated that there were obvious differences between obese mice and normal weight mice in their physical index and metabolic status.

Oxidative stress occurred in ob/ob mice, as indicated by decreased SOD activity and elevated MDA levels

To evaluate the relationship between obesity and oxidative stress, we measured SOD activity and the serum level of lipid peroxidation (MDA production). As shown in Fig. 1A, the activity of SOD was lower in ob/ob mice than in the C57BL/6J group $(201.80 \pm 27.34 \mathrm{U} / \mathrm{ml}$ vs. $233.67 \pm 29.78 \mathrm{U} / \mathrm{ml}, p<0.01)$, while the level of MDA (Fig. 1B) was higher $(3.56 \pm 0.19 \mu \mathrm{mol} / \mathrm{l}$ vs. $2.55 \pm 0.26 \mu \mathrm{mol} / \mathrm{l}, p<0.01$ ). This demonstrated that there was systemic oxidative stress in obese status.

Augmented Rac1 expression and oxidative stress existed in pancreatic tissue in ob/ob mice

All mice were killed at the age of eight weeks at the end of the experiment, and their pancreases were carefully removed for further study. Rac1 expression in pancreatic tissue was assessed by western blot. As shown in Fig. 2A, compared with C57BL/6J, Rac1 expression in pancreatic tissue was significantly increased in ob/ob mice. This finding was confirmed by immunohistochemistry (Fig. 3A): compared with C57BL/6J mice, the staining in the pancreatic island of ob/ob mice was even darker, indicating that obese status is characterized by augmented Rac1 expression in pancreatic islets. DHE staining was performed to detect superoxide generation in the pancreas. Ultimately, more superoxide free radical generation was triggered in ob/ob mice, as indicated by positive intense red fluorescent staining (Fig. 3B).

Table 1. Physical measurement, metabolic index, and insulin sensitivity of the mice involved in this study

\begin{tabular}{lllllll}
\hline & C57BL/6J & $o b / o b$ & Pvalue & NSC ob/ob & PBS ob/ob & Pvalue \\
\hline Weight $(\mathrm{g})$ & $20.31 \pm 0.76$ & $46.34 \pm 2.29$ & 0.001 & $45.11 \pm 2.10$ & $45.91 \pm 1.51$ & 0.665 \\
Lee's index & $320 \pm 12.9$ & $365.2 \pm 13.6$ & 0.031 & $346.5 \pm 13.8$ & $359.3 \pm 14.7$ & 0.431 \\
FBG $(\mathrm{mM})$ & $7.85 \pm 1.12$ & $12.0 \pm 3.50$ & 0.011 & $10.1 \pm 1.89$ & $13.80 \pm 4.49$ & 0.025 \\
INS $(\mu \mathrm{U} / \mathrm{ml})$ & $10.74 \pm 1.13$ & $18.68 \pm 3.51$ & 0.001 & $17.81 \pm 3.33$ & $19.58 \pm 3.86$ & 0.051 \\
HOMA & $3.74 \pm 0.89$ & $10.47 \pm 1.45$ & 0.001 & $7.20 \pm 1.05$ & $9.62 \pm 0.95$ & 0.048 \\
TG $(\mathrm{mM})$ & $0.77 \pm 0.08$ & $1.26 \pm 0.10$ & 0.028 & $1.14 \pm 0.09$ & $1.37 \pm 0.09$ & 0.139 \\
TC $(\mathrm{mM})$ & $2.65 \pm 0.21$ & $3.68 \pm 0.31$ & 0.023 & $3.39 \pm 0.24$ & $3.57 \pm 0.28$ & 0.232 \\
\hline
\end{tabular}

Data are means \pm SD. NSC ob/ob: male ob/ob mice that were intraperitoneally injected with NSC23766 for 2 weeks; PBS ob/ob: ob/ob mice that received an equal volume of phosphate buffer solution (PBS) for 2 weeks; Lee's index=weight $1 / 3 \times 10^{3} /$ length. FBG, fasting blood glucose; INS, insulin; HOMAIR, homeostasis model of insulin resistance: $\mathrm{HOMA}_{\mathrm{IR}}=\left(\mathrm{I}_{0} \times \mathrm{G}_{0}\right) / 22.5$ (insulin in $\mu \mathrm{U} / \mathrm{ml}$ and glucose in $\mathrm{mM}$ ); TC, total cholesterol; TG, triglyceride 
Fig. 1. Oxidative stress occurred in ob/ob mice, and NSC23766 can suppress oxidative stress. 4-week-old C57BL/6J mice (10) and ob/ob mice (30) were selected and kept until 8 weeks old. Among them, twenty ob/ob mice were randomized into two groups that were given NSC23766 (2.5 mg. $\mathrm{kg}^{-1}$. day ${ }^{-1}$, i.p.) or an equal volume of PBS for 2 weeks. The serum malondialdehyde (MDA) level and superoxide dismutase (SOD) activity were measured. The SOD activity (Fig. 1A) was lower, in ob/ob mice than that in C57BL/6J group $(201.80 \pm 27.34 \mathrm{U} / \mathrm{ml}$ vs. $233.67 \pm 29.78 \mathrm{U} / \mathrm{ml}$, $p<0.01$ ), while the MDA level (Fig. 1B) was higher in ob/ob mice $(3.56 \pm 0.19 \mu \mathrm{mol} / \mathrm{l}$ vs. $2.55 \pm 0.26 \mu \mathrm{mol} / \mathrm{l}$, $p<0.01$ ). After the intervention, the SOD activity (Fig. $1 \mathrm{~A})$ in the NSC ob/ob group was elevated compared with the PBS ob/ob group $(220.34 \pm 26.68 \mathrm{U} / \mathrm{ml}$ vs. $209.11 \pm 26.82 \mathrm{U} / \mathrm{ml}, p<0.01$ ), while the MDA level (Fig. 1B) was lower $(3.02 \pm 0.18 \mu \mathrm{mol} / \mathrm{l}$ vs. $3.43 \pm 0.22$ $\mu \mathrm{mol} / \mathrm{l}, p<0.05$ ). ${ }^{* *} p<0.01 \mathrm{C} 57 \mathrm{BL} / 6 \mathrm{~J} v s . \mathrm{ob} / \mathrm{ob}$ and NSC ob/ob vs. PBS ob/ob, ${ }^{*} p<0.05$ NSC ob/ob vs. PBS ob/ob.

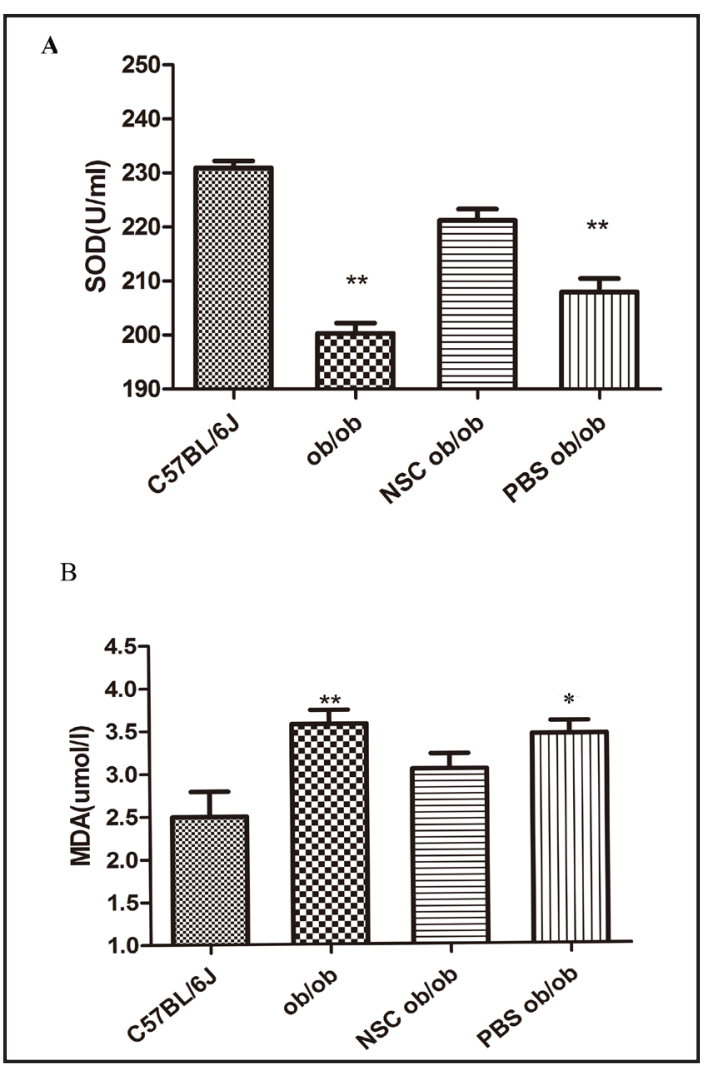

The Rac1 inhibitor-NSC23766 decreased the levels of fasting blood glucose and insulin resistance and ameliorated $\beta$ cell function

We injected the specific Rac1 inhibitor-NSC23766 into ob/ob mice for 14 days and detected any differences between the NSC ob/ob and the PBS ob/ob group. The results indicated that NSC23766 decreased the level of FBG and the degree of insulin resistance (Table 1). Meanwhile, no significant differences in characteristics such as weight, Lee's index, insulin, TC and TG were found between the NSC ob/ob group and PBS ob/ob group (Table 1).

The Rac1 inhibitor NSC23766 suppressed oxidative stress in ob/ob mice

SOD activity was higher in NSC ob/ob mice than in PBS ob/ob $(220.34 \pm 26.68 \mathrm{U} / \mathrm{ml}$ vs. $209.11 \pm 26.82 \mathrm{U} / \mathrm{ml}, p<0.01)$, while the MDA level was lower $(3.02 \pm 0.18 \mu \mathrm{mol} / \mathrm{l} \mathrm{vs} .3 .43 \pm 0.22$ $\mu \mathrm{mol} / \mathrm{l}, p<0.05$ ), which meant that the repression of Rac1 interferes with oxidative stress to some extent (Fig. 1).

NSC23766 decreased Rac1 expression and oxidative stress in pancreatic tissue in ob/ob mice

After ob/ob mice were treated with NSC23766 for 2 weeks, the Rac1 expression in the pancreas decreased, as shown by western blot (Fig. 2B). Then, we used a histological method to confirm the previous finding. As is shown in Fig. 3A, the staining in the pancreatic islands of NSC ob/ob mice was lighter compared with the untreated PBS ob/ob mice, indicating a decrease in Rac1 expression. Simultaneously, oxidative stress in the pancreas was relieved by the inhibition of Rac1, as indicated by the subdued red fluorescent DHE staining (Fig. 3B).

Oxidative stress in NIT-1 $\beta$ cells induced by hyperglycemia and/or hyperlipidemia was accompanied by the activation of NADPH oxidase and the translocation of Rac1 to the cellular membrane

To mimic the oxidative status, we added glucose $(20 \mathrm{mM})$, palmitic acid $(100 \mu \mathrm{M})$, and both into the cell suspension. The fluorescence in NIT- $1 \beta$ cells was determined by a 
Fig. 2. ob/ob mice showed elevated Rac1 expression in pancreatic tissue, which could be decreased by NSC23766. Rac1 expression in pancreatic tissue was assessed by western blot. Compared with C57BL/6], Rac1 expression in pancreatic tissue was significantly increased in ob/ob mice (Fig. 2A). After the ob/ob mice were treated by NSC23766 for 2 weeks, the expression of Rac1 obviously declined (Fig. 2 B). ${ }^{* *} p<0.01$ C57BL/6J vs. ob/ob, ${ }^{*} p<0.05$ NSC ob/ob vs. PBS ob/ob.

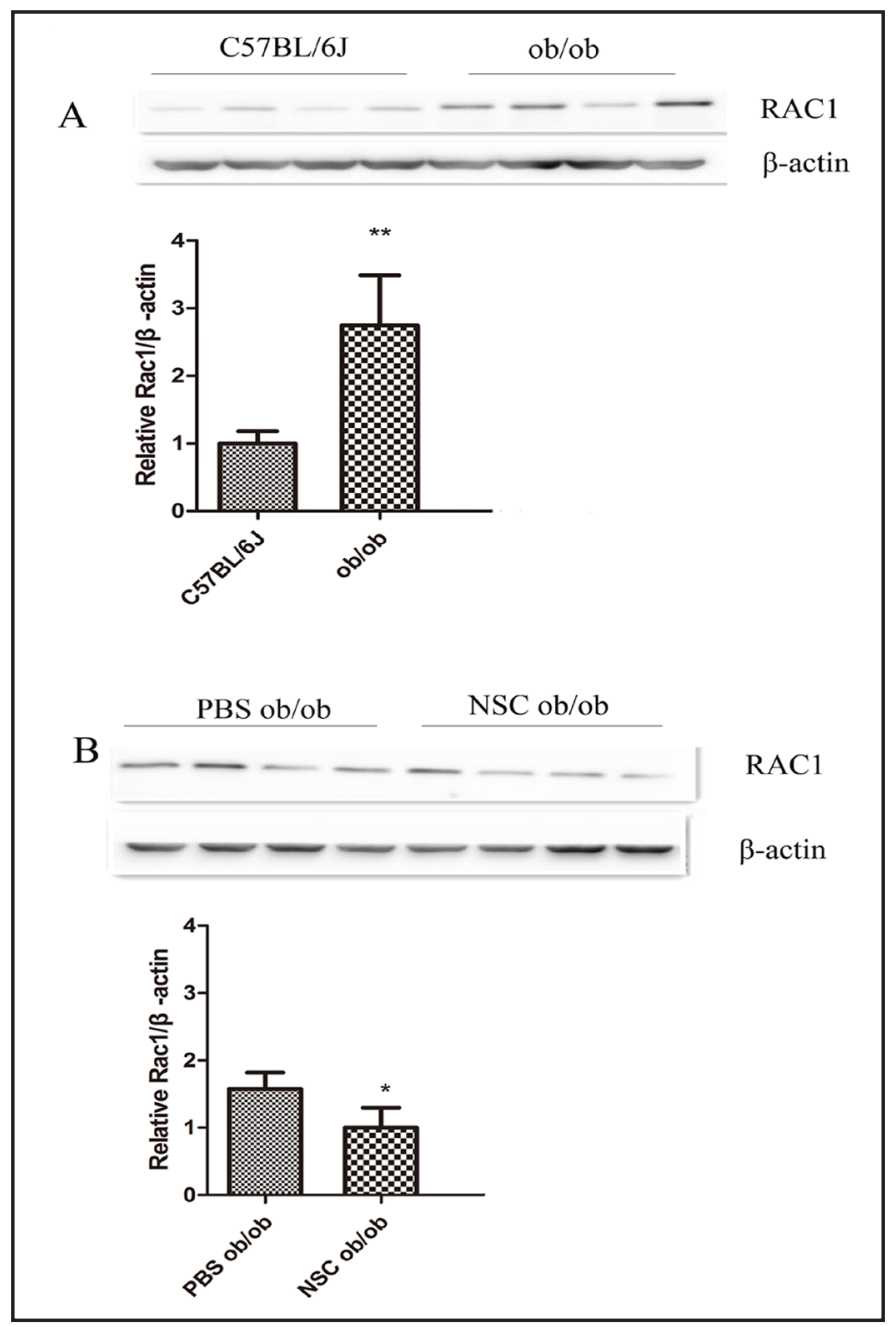

flow cytometer. Compared with the blank control group, ROS was increased by $19.6 \%$ in the hyperglycemia group, $15.1 \%$ in hyperlipidemia group and $24.1 \%$ in the group incubated with both compounds, as shown in Fig. 4A. Meanwhile, exposure of these cells to elevated glucose (glucotoxicity), fatty acids (lipotoxicity) or both (glucolipotoxicity) resulted in a significant increase in the NOX enzyme activity (Fig. 4C). To find out the role of Rac1 in this process, we applied the indirect immunofluorescence method to observe changes in Rac1 distribution. As shown in Fig. 5, Rac1's translocation to the cellular membrane was promoted in the hyperglycemic and/or hyperlipidemic state, which might help to anchor cytosolic p67phox to the membrane for the assembly of active NADPH oxidase, leading to the activation of NADPH oxidase and superoxide generation.

Insulin mRNA in NIT-1 $\beta$ cells was lessened in the oxidative state induced by hyperglycemia and/or hyperlipidemia

We incubated NIT-1 $\beta$ cells with $20 \mathrm{mM}$ glucose, $100 \mu \mathrm{M}$ palmitic acid, or both, then extracted the insulin mRNA in the cells. We found that insulin mRNA in $\beta$ cell was decreased by $74.1 \%$ in the hyperglycemia group, $57.1 \%$ in the hyperlipidemia group and $62.8 \%$ in the group incubated with both substances compared with the blank control group (Fig. 6).

Attenuation of Rac1 could alleviate the oxidative stress and activation of NADPH oxidase induced by hyperglycemia and/or hyperlipidemia in NIT-1 $\beta$ cells

To further demonstrate the role of Rac1 in the generation of ROS, a highly selective Rac1 inhibitor, NSC23766, was added to the cell suspension of hyperglycemia and/or 


\section{Cellular Physiology Cell Physiol Biochem 2015;35:2135-2148

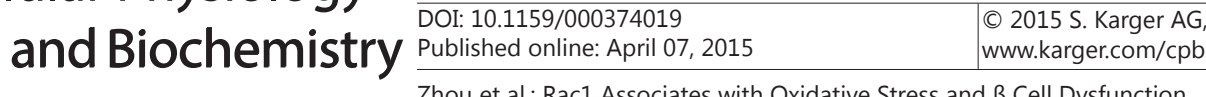

Fig. 3. Obesity led to augmented Rac1 expression and oxidative stress in pancreatic tissue, which could be attenuated by NSC23766. Rac1 expression in pancreatic tissue was confirmed by immunohistochemistry. All pictures were taken with confocal microscopy. In Fig. 3A, compared with C57BL/6J, the staining in pancreatic islands of ob/ ob mice was darker, which indicted that obese status was companied by augmented Rac1 expression in islets. After the administration of NSC for 2 weeks, the staining in the pancreatic islands of NSC ob/ob mice became lighter than those of the untreated PBS ob/ob mice. DHE staining was performed to detect superoxide generation in pancreatic tissue. In Fig. 3B, more superoxide free radical generation was triggered in ob/ ob mice compared with C57BL/6J mice, as indicated by positive red fluorescent staining. After the administration of NSC23766, the red fluorescent staining of pancreatic tissues in ob/ob mice declined.

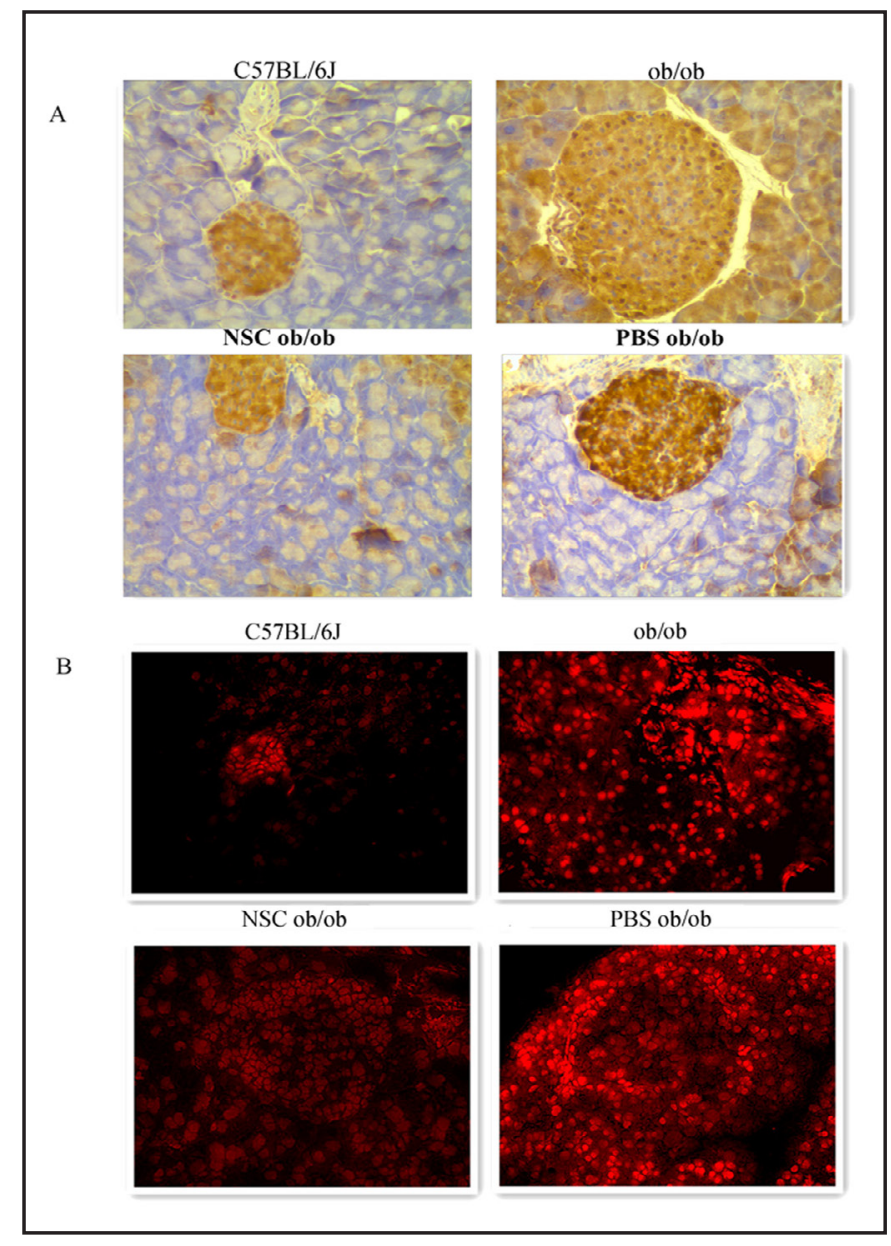

hyperlipidemia cells overnight. As demonstrated in Fig. 4B, we found that the ROS in NIT$1 \beta$ cells was significantly attenuated, which proved that the activation of Rac1 had taken part in the generation of oxidative stress induced by hyperglycemia and/or hyperlipidemia in NIT-1 $\beta$ cells. Inhibiting Rac1 activity decreases the oxidative stress to a certain extent. Meanwhile, the co-provision of NSC23766 attenuated the ability of glucolipotoxic condition to stimulate NADPH oxidase activity (Fig. 4C). The differences in cellular ROS induced by NSC23766 are summarized and displayed in Fig. 4D.

Suppression of Rac1 could reduce its translocation to the cellular membrane

Rac1 activation is required to anchor cytosolic p67phox to the membrane for the assembly of active NADPH oxidase, leading to superoxide generation. In the present study, we found that Rac1 translocation to the cellular membrane was promoted under hyperglycemic and/or hyperlipidemic conditions. Then, we treated the cells with NSC23766 and used an indirect immunofluorescence method to observe the changes in the cells. We found that the fluorescence was mostly observed in the cytoplasm, as illustrated in Fig. 5, which indicates that NSC23766 could inhibit Rac1 translocation to the cellular membrane.

The impairment of $\beta$ cell function induced by oxidative stress can be ameliorated by inhibiting Rac1

To further demonstrate the role of Rac1 in $\beta$ cell dysfunction, we used the Rac1 inhibitor NSC23766 to treat hyperglycemia and/or hyperlipidemia cells and found that the insulin mRNA in NIT-1 $\beta$ cells was significantly higher (Fig. 6), which demonstrated that the overexpression of Rac1 was associated with the decline of $\beta$ cell function in obesity and that inhibiting Rac1 should improve $\beta$ cell function. 

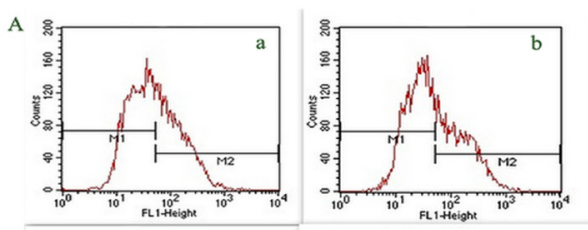

B
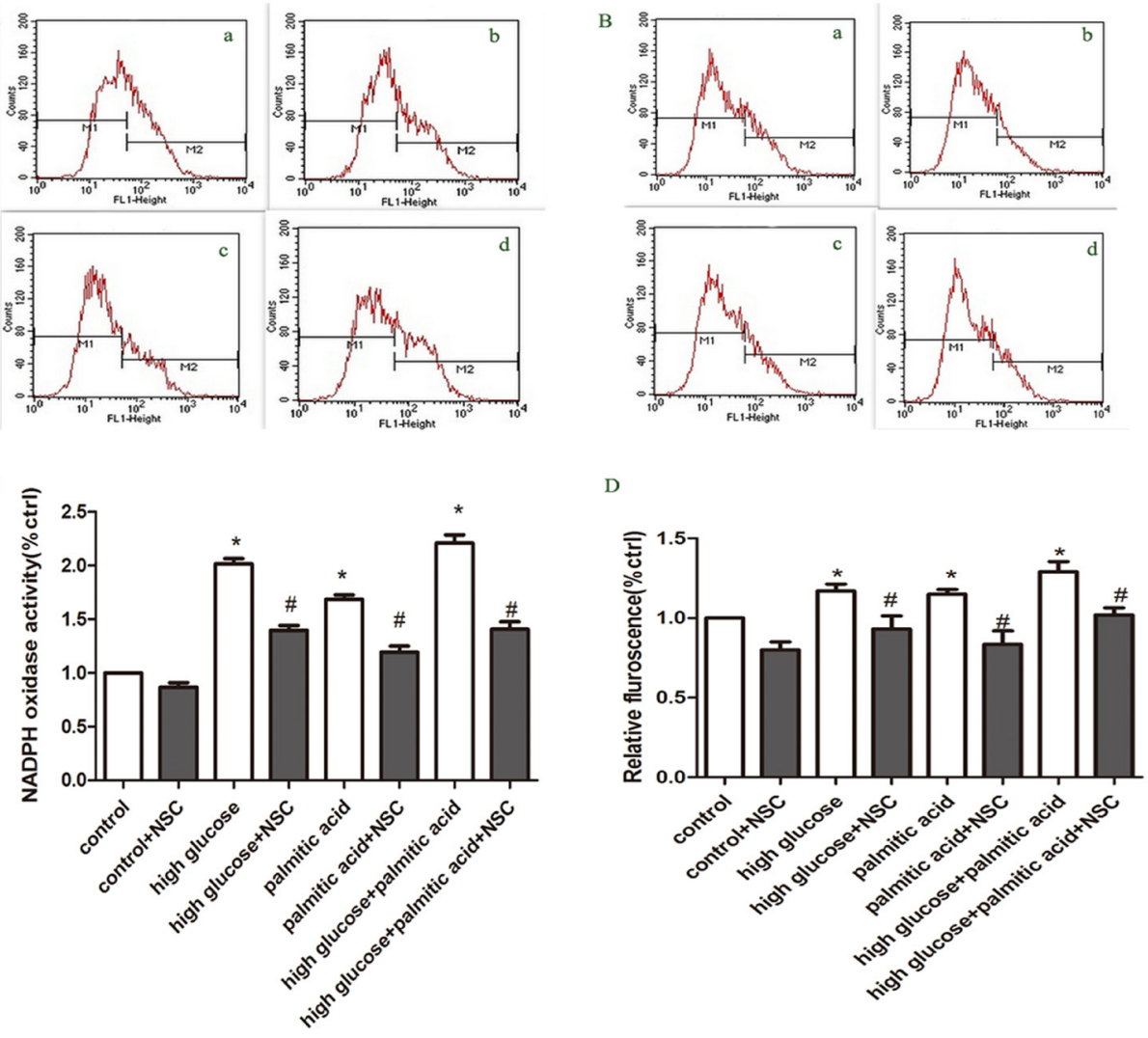

Fig. 4. Oxidative stress in NIT-1 $\beta$ cells can be induced by hyperglycemia and/or hyperlipidemia, and attenuation of Rac1 can alleviate oxidative stress induced by hyperglycemia and/or hyperlipoidemia in NIT-1 $\beta$ cells. NIT-1 $\beta$ cells were incubated with additional $20 \mathrm{mM}$ glucose, $100 \mu \mathrm{M}$ palmitic acid, or both, and fluorescence was determined by a flow cytometer. Compared with the blank control group, ROS increased by $19.6 \%$ in the hyperglycemia group, $15.1 \%$ in the hyperlipidemia group and $24.1 \%$ in the group incubated with both, as can be observed in Fig. 4A (a. control group; b. hyperglycemia group; c. hyperlipidemia group; d. hyperglycemia and hyperlipidemia group). Exposure of these cells to elevated glucose (glucotoxicity), fatty acids (lipotoxicity) or both (glucolipotoxicity) resulted in a significant increase in the NOX enzyme activity (Fig. 4C). NSC23766 (20 $\mu \mathrm{M})$ was added to the cell suspension overnight, and then the fluorescence in NIT-1 $\beta$ cells was determined using a flow cytometer. As demonstrated in Fig. 4B, ROS in NIT-1 $\beta$ cells was significantly attenuated (a. control+NSC group; b. hyperglycemia+NSC group; c. hyperlipidemia+NSC group; d. hyperglycemia and hyperlipidemia+NSC group). Meanwhile, co-provision of NSC23766 attenuated the ability of the glucolipotoxic condition to stimulate NADPH oxidase activity (Fig. 4C).The differences of ROS induced by NSC23766 are summarized and displayed in Fig. 4D. ${ }^{*} p<0.01 v s$.control, $\# p<0.05$ NSC+ hyperglycemia/hyperlipidemia/both vs. hyperglycemia/ hyperlipidemia/both.

\section{Discussion}

The primary purpose of the present study was to clarify the relationship between Rac1 expression and activation, the generation of superoxides and $\beta$ cell dysfunction in obesity. Here, we provided novel experimental evidence showing that the increased expression and activation of Rac1 contributed to oxidative stress, both systemically and locally, in the pancreas in obese mice, which might also imply a participatory role in $\beta$ cell dysfunction.

We previously reported that overweight adolescents displayed discrepancies in glycometabolism, lipid metabolism, and insulin sensitivity compared to normal weight adolescents; increased oxidative stress existed in overweight adolescents and was primarily 


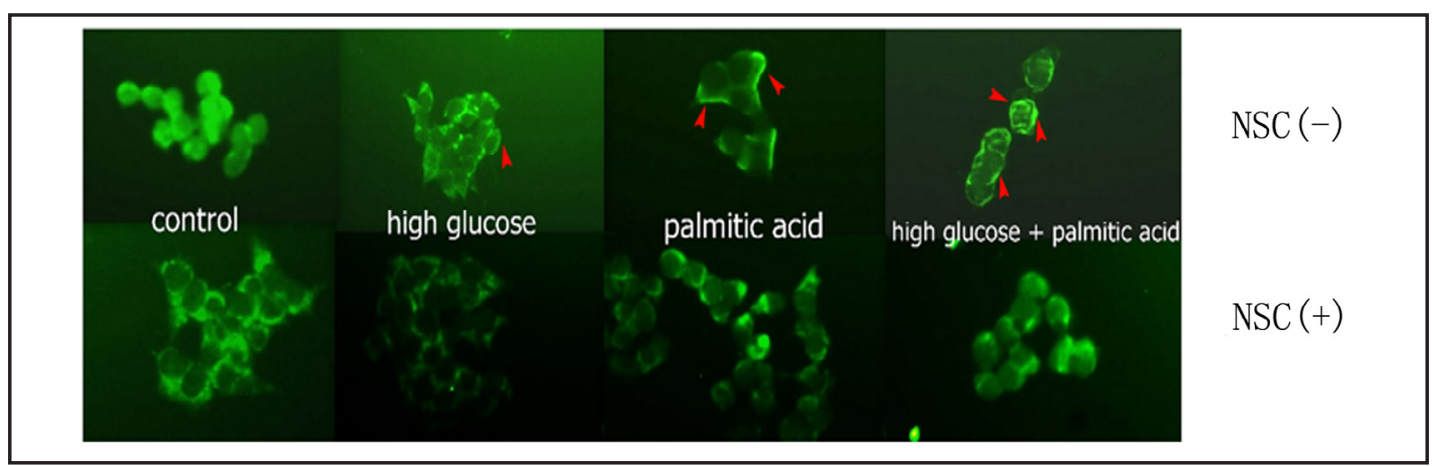

Fig. 5. Oxidative stress induced by hyperglycemia and/or hyperlipidemia in NIT-1 $\beta$ cells was accompanied by the translocation of Rac1 to the cellular membrane; suppression of Rac1 can inhibit the translocation. Indirect immunofluorescence was applied to observe the changes in the cells under hyperglycemic and/or hyperlipidemic conditions. Compared with the blank control group, fluorescence gathered around the edge of the cells in the high glucose group, the palmitic acid group and the group incubated with both, which indicated that Rac1 translocated to the cellular membrane. After the treatment with NSC23766, the fluorescence was scattered in the cytoplasm, which indicates that NSC23766 can inhibit the translocation of Rac1 to the cellular membrane.

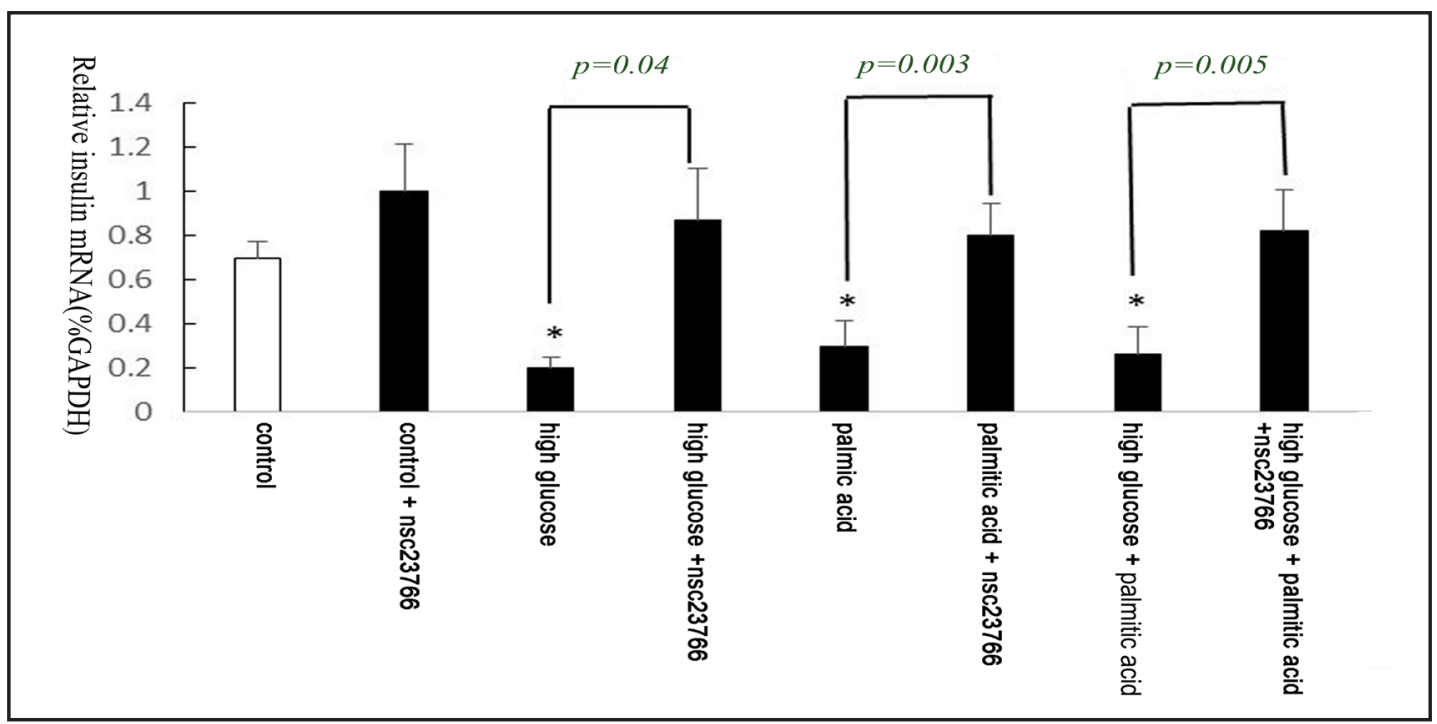

Fig. 6. Insulin mRNA in NIT-1 $\beta$ cells was decreased in the oxidative state induced by hyperglycemia and/ or hyperlipidemia, which could be ameliorated by inhibiting Rac1. We incubated NIT-1 $\beta$ cells with 20 mM glucose, $100 \mu \mathrm{M}$ palmitic acid, or both, then isolated the insulin mRNA. As is shown in Fig. 6, insulin mRNA in $\beta$ cells decreased by $74.1 \%$ in the hyperglycemia group, $57.1 \%$ in the hyperlipidemia group and $62.8 \%$ in the group incubated with both; then, the Rac1 inhibitor NSC23766 was used to treat the cells, which caused insulin mRNA to increase significantly.

caused by enhancing Rac1 expression and the augmented activity of NADPH oxidase [9]. In the present study, ob/ob mice displayed similar abnormal glucose and lipid metabolism and oxidative stress, which were highly consistent with our previous studies in overweight adolescents [14] and clinical studies that have indicated that obese patients are more susceptible to impaired glucose tolerance and an early-onset diabetes [15]. Recent studies by Gurzov et al. have reported that oxidative stress accompanying obesity inactivates proteintyrosine phosphatases (PTPs) in the liver, thus activating select signaling pathways that exacerbate disease progression [16]. Mai A et al. have studied Nox2/ApoE double-knockout mice eating a high-fat diet and found that Nox2-derived oxidative stress plays an important role in the pathogenesis of dietary obesity-associated metabolic syndrome and endothelial 
dysfunction. Recent studies by Sukumar and coworkers have probed the potential regulatory roles of Nox in models of human insulin resistance [17]. They observed significantly higher levels of superoxides in insulin-resistant endothelial cells. Pharmacological inhibition using gp91ds-tat or siRNA-mediated knockdown of Nox2 significantly attenuated superoxide generation in these cells. Further supporting a role for Nox2 in the onset of these metabolic defects, these authors reported that a double transgenic mouse model [i.e., with endothelialspecific insulin resistance and the deletion of Nox2] exhibited attenuated superoxide production and improved vascular function, corroborating our data suggesting the potential relation of oxidative stress and the duress imposed by glucolipotoxicity in obesity.

$\beta$ cell dysfunction plays an essential role in the pathogenesis of diabetes [18]. Increased intracellular generation of ROS has been implicated in the pathology of metabolic diseases. Accumulating evidence suggests Nox as the principal source for cellular ROS in humans and as one of the "culprits" for the induction of cellular damage culminating in the onset of diabetes and its complications [19]. Nox is a highly regulated membrane-associated protein complex that promotes the one-electron reduction of oxygen to a superoxide anion by the oxidation of cytosolic NADPH. The Nox holoenzyme consists of membrane and cytosolic components. The membrane-associated catalytic core consists of gp91phox and p22phox, and the cytosolic regulatory core includes p47phox, p67phox, p40phox, and Rac1 [20]. Under stimulatory conditions, the cytosolic components translocate to the membrane fraction for association with the catalytic core for Nox2 holoenzyme assembly and catalytic activation. Rac1 is necessary to anchor cytosolic p67phox to the membrane for the assembly of active Nox, inducing superoxide generation [21].

Several plausible mechanisms have been proposed for the generation of ROS and associated oxidative stress in a variety of cell types, including the islet $\beta$ cell $[22,23]$. Ismail Syed et al. have reported a significant increase in the subunit expression and activation of Nox2 in islets derived from the Zucker Diabetic Fatty rat, a model for type 2 diabetes [24]. Yuan and associates noted high levels of ROS generation and decreased insulin content in islets from Sprague-Dawley rats following a 24-week high fat feeding [25]. They also reported increased ROS generation and a reduction in insulin content following the exposure of insulin-secreting NIT-1 cells to high glucose concentrations in vitro. Recent studies have demonstrated that local NADPH oxidase activity in the pancreas increased in diabetes $[26,27]$. As is shown in the current study, in addition to abnormal metabolic status, insulin resistance, and systemic oxidative stress in ob/ob mice, we also found that the ROS production and the expression of Rac1 were significantly increased in the pancreas of ob/ob mice. This result conforms with our former study that Rac1 might play a key role in obesity-related changes [9] and was further confirmed by the specific inhibition of Rac1. After 14 days administration of NSC23766, a specific activity inhibitor of Rac1, the serum levels of fasting glucose and the degree of insulin resistance declined along with the decrease of ROS production and Rac1 expression in pancreas, which suggested that the suppression of Rac 1 could decline the oxidative stress level, ameliorate $\beta$ cell function, and further improve glycometabolism. There were no significant differences in characteristics such as weight, Lee's index, TC and TG after the treatment of NSC23766, suggesting that the role of oxidative stress induced by Rac1 on glycometabolism might be independent of changes in body weight and physical composition.

It is known that an important step for the assembly of active Nox is the translocation of cytosolic subunits (p67phox, p47phox, and Rac1) to membrane subunits (gp91phox and p22phox) [28, 29]. Rac1 activation (the GTP-bound conformation) leads to its association with p67phox, triggering the translocation of the Rac1-p67phox dimer to the membrane. Recent evidence suggests novel regulatory roles for specific guanine nucleotide exchange factors (GEFs) for Rac1 in attaining its GTP-bound active configuration [30]. There is evidence showing that oxidative stress is enhanced in the mouse heart failure model of hypertensive heart disease (HHD), Rac1 activity is upregulated in the heart of HHD mice, and cardiac function is significantly impaired at the same time [31]. Lopez-Haber et al. found that ErbB-driven Rac1 activation in breast cancer cells proceeded independently of the 


\section{Cellular Physiology Cell Physiol Biochem 2015;35:2135-2148 \begin{tabular}{l|l} 
and Biochemistry Published online: April 07, 2015 & $\begin{array}{l}\text { C) 2015 S. Karger AG, Basel } \\
\text { www.karger.com/cpb }\end{array}$ \\
\hline
\end{tabular} \\ Zhou et al.: Rac1 Associates with Oxidative Stress and $\beta$ Cell Dysfunction}

Jak2 pathway, and cucurbitacin I inhibited Rac1 activation in breast cancer cells by a ROSmediated mechanism [32]. To discover the cellular and molecular mechanism connecting Rac 1 to oxidative stress, we extended our analyses to NIT-1 $\beta$ cells by applying hyperglycemic and/or hyperlipoidemic conditions to mimic the oxidative stress status. The analysis of subcellular localization of Rac1 by confocal immunofluorescence microscopy showed that Rac1 presented a cytoplasmic distribution primarily in normal $\beta$ cells, but it translocated to the membrane in response to hyperglycemia and/or hyperlipoidemia stimulation accompanied by enhanced ROS production, suggesting excessive activation of Rac1 under glucotoxicity, lipotoxicity or glucolipotoxicity. Moreover, selective inhibition of Rac1 could alleviate oxidative stress induced by hyperglycemia and/or hyperlipoidemia in NIT-1 $\beta$ cells. We demonstrated that the activation of Rac1 played a role in ROS production in NIT-1 $\beta$ cells. Similar studies by Subasinghe and associates have demonstrated contributory roles for Nox in cytokine-induced metabolic dysfunction of the islet $\beta$ cell and a significant reduction in proinflammatory cytokine-induced Rac1 activation and Nox-mediated ROS generation by inhibitors of Rac1 [33]. Furthermore, Syed and associates have reported a marked increase in Rac1 activation and the generation of superoxides and lipid peroxides in INS-1 832/13 cells following exposure to palmitate [7]. These observations support the hypothesis that metabolic dysfunction of the islet $\beta$ cell induced by hyperglycemia and/or hyperlipoidemia requires the intermediacy of the Rac1-Nox signaling pathway.

Our subsequent data revealed the role of Rac1 and ROS in $\beta$ cell function variation by examining insulin mRNA in NIT-1 $\beta$ cells. Recent studies suggest that ROS derived from Nox play regulatory "second-messenger" roles in GSIS [30, 34]. Indeed, previous studies showed that Rac1 plays a pivotal role in insulin-stimulated glucose uptake in skeletal muscle, which is mediated by GLUT4 translocation to the plasma membrane [35]. In addition to the positive modulatory roles for ROS in GSIS, recent evidence also implicates negative modulatory roles for ROS in the induction of oxidative stress and metabolic dysregulation of islet $\beta$ cells under the duress of glucolipotoxicity, cytokines, and ceramide [8]. The generation of ROS in these experimental conditions is largely due to the activation of Nox because inhibition of Rac1 or Nox activation markedly attenuated the deleterious effects of these stimuli. Recently, Hwaiz et al. found that Rac 1 activity was increased in lungs from septic animals, and administration of NSC23766 markedly reduced sepsis-triggered neutrophil infiltration, edema formation, and tissue damage in the lung [36]. Similar results were observed in our study, which found that the impaired $\beta$ cell function could be recovered partly by inhibiting Rac1 with the administration of NSC23766, which also proved that over-activation of Rac1 contributed to the decline of $\beta$ cell function in the progress of obesity from the other side. On the basis of the existing information, Kowluru et al. [24] proposed the following model for Nox-mediated induction of $\beta$ cell dysfunction in diabetes as the Rac1-Nox-ROS signaling pathway: exposure of isolated $\beta$ cells to glucolipotoxic conditions or islets derived from the diabetic individuals results in increased activation of Rac1 and Nox. The consequent generation of ROS and the associated oxidative stress, in turn, promote the activation of JNK1/2 and mitochondrial dysregulation. Our findings demonstrated this signaling pathway also exists in obesity.

There are some limitations to our study. First, we performed western blots on pancreas tissue, not on isolated islets; thus, some confounding factors from the exocrine portion cannot be ruled out. However, we observed the expression of Rac1 in islets by immunohistochemical methods at the same time, indicating the level of Rac1 semiquantitatively. Second, although we used a well-defined and acceptable cell-line (NIT-1) to study the function of $\beta$ cells, it would be preferable to also perform the study on endocrine function in primary beta-cells. Third, if C57 mice were also treated with NSC, the result would be more complete and convincing. Fourth, we used hyperlipidemic conditions to mimic the status in obese individuals and found activation of NADPH oxidase and translocation of Rac1 under hyperlipidemia. Furthermore, insulin mRNA in NIT-1 $\beta$ cells was decreased. Suppression of Rac1 by NSC could reduce Rac1 translocation and $\beta$ cell dysfunction in hyperlipidemia. However, we have not observed this phenomenon in vivo. The reason for NSC's lack of effect on TG levels needs to be determined in further studies. 


\section{Cellular Physiology Cell Physiol Biochem 2015;35:2135-2148 \begin{tabular}{ll|l} 
and Biochem 10.1159/000374019 & $\begin{array}{l}\text { O 2015 S. Karger AG, Basel } \\
\text { www.karger.com/cpb }\end{array}$ \\
\hline
\end{tabular} \\ Zhou et al.: Rac1 Associates with Oxidative Stress and $\beta$ Cell Dysfunction}

In conclusion, increased intracellular generation of ROS has been implicated in the pathology of metabolic and neurodegenerative diseases. Nox plays contributory roles in the induction of cellular damage culminating in the onset of metabolic disorders. Our findings suggested that Rac1 might contribute to oxidative stress in systemic and pancreatic levels in obesity. The excessive activation and expression of Rac1 in obesity were associated with $\beta$ cell dysfunction through ROS production, disturbing the metabolic homeostasis, and this might be one of the latent mechanisms promoting the onset of diabetes. Targeting Rac1Nox-ROS signaling represents a valuable therapeutic strategy to obesity and diabetes. Future investigations will focus on the development of specific inhibitors for these signaling steps to halt Nox activation and cellular defects/damage in obesity, diabetes and other disorders.

\section{Disclosure Statement}

No potential conflicts of interest relevant to this article were reported.

\section{Acknowledgments}

This project was funded by the Science Foundation of Beijing (7092090), and Chinese Medical Association Scientific Research Foundation (13060970482). S.Z. participated in the execution of experiments and the analysis of experimental data and wrote the manuscript. D.Y. participated in the execution of experiments and the analysis of experimental data. S.N. and M.L. contributed to the execution of the cellular experiments. L.J. was involved in conceiving the experiments/research program. L.H. and H.Z. contributed to the execution of the zoological experiments. M.S. designed the research program, reviewed and edited the manuscript, and contributed to discussion. M.S. is the guarantor of this work and, as such, has full access to all the data in the study and takes responsibility for the integrity of the data and the accuracy of the data analysis. The first two authors contributed equally to this work. The authors thank the Beijing Institute of Geriatrics, Ministry of Health for technical assistance.

\section{References}

1 Tateya S, Kim F, Tamori Y: Recent advances in obesity-induced inflammation and insulin resistance. Front Endocrinol (Lausanne) 2013;4:93.

2 You T, Arsenis NC, Disanzo BL, Lamonte MJ: Effects of exercise training on chronic inflammation in obesity: current evidence and potential mechanisms. Sports Med 2013;43:243-256.

3 Aguilar Cordero MJ, Gonzalez Jimenez E, Sanchez Perona J, Padilla Lopez CA, Alvarez Ferre J, Ocete Hita E, Rizo Baeza M, Guisado Barrilao R, Garcia Rivas F: [Obesity and its relation with markers of inflammation and erythrocyte fatty acids in a group of overweight adolescents]. Nutr Hosp 2012; 27:161-164.

4 Du Z, Hu Y, Yang Y, Sun Y, Zhang S, Zhou T, Zeng L, Zhang W, Huang X, Kong W, Zhang H: NADPH oxidasedependent oxidative stress and mitochondrial damage in hippocampus of D-galactose-induced aging rats. J Huazhong Univ Sci Technolog Med Sci 2012;32:466-472.

5 Kleniewska P, Piechota A, Skibska B, Goraca A: The NADPH oxidase family and its inhibitors. Arch Immunol Ther Exp (Warsz) 2012;60:277-294.

6 Zhao R, Le K, Moghadasian MH, Shen GX: Regulatory role of NADPH oxidase in glycated LDL-induced upregulation of plasminogen activator inhibitor-1 and heat shock factor-1 in mouse embryo fibroblasts and diabetic mice. Free Radic Biol Med 2013;61C:18-25.

7 Syed I, Jayaram B, Subasinghe W, Kowluru A: Tiam1/Rac1 signaling pathway mediates palmitate-induced, ceramide-sensitive generation of superoxides and lipid peroxides and the loss of mitochondrial membrane potential in pancreatic beta-cells. Biochem Pharmacol 2010;80:874-883. 


\section{Cellular Physiology Cell Physiol Biochem 2015;35:2135-2148 \begin{tabular}{l|l} 
and Biochemistry Published online: April 07, 2015 & $\begin{array}{l}\text { C) 2015 S. Karger AG, Basel } \\
\text { www.karger.com/cpb }\end{array}$ \\
\hline
\end{tabular} \\ Zhou et al.: Rac1 Associates with Oxidative Stress and $\beta$ Cell Dysfunction}

8 Kowluru A: Friendly, and not so friendly, roles of Rac1 in islet beta-cell function: lessons learnt from pharmacological and molecular biological approaches. Biochem Pharmacol 2011;81:965-975.

9 Sun M, Huang X, Yan Y, Chen J, Wang Z, Xie M, Li J: Rac1 is a possible link between obesity and oxidative stress in Chinese overweight adolescents. Obesity 2012;20:2233-2240.

10 Shen E, Li Y, Li Y, Shan L, Zhu H, Feng Q Arnold JM, Peng T: Rac1 is required for cardiomyocyte apoptosis during hyperglycemia. Diabetes 2009;58:2386-2395.

11 Shah GN, Price TO, Banks WA, Morofuji Y, Kovac A, Ercal N, Sorenson CM, Shin ES, Sheibani N: Pharmacological inhibition of mitochondrial carbonic anhydrases protects mouse cerebral pericytes from high glucose-induced oxidative stress and apoptosis. J Pharmacol Exp Ther 2013;344:637-645.

12 Hwang SY, Siow YL, Au-Yeung KK, House J, O K: Folic acid supplementation inhibits NADPH oxidasemediated superoxide anion production in the kidney. Am J Physiol Renal Physiol 2011;300:F189-198.

13 Geetha R, Yogalakshmi B, Sreeja S, Bhavani K, Anuradha CV: Troxerutin suppresses lipid abnormalities in the heart of high-fat-high-fructose diet-fed mice. Mol Cell Biochem 2014;387:123-134.

14 Sun MX, Huang XQ, Yan Y, Li BW, Zhong WJ, Chen JF, Zhang YM, Wang ZZ, Wang L, Shi XC, Li J, Xie MH: Onehour after-school exercise ameliorates central adiposity and lipids in overweight Chinese adolescents: a randomized controlled trial. Chin Med J (Engl) 2011;124:323-329.

15 Mencarelli M, Zulian A, Cancello R, Alberti L, Gilardini L, Di Blasio AM, Invitti C: A novel missense mutation in the signal peptide of the human POMC gene: a possible additional link between early-onset type 2 diabetes and obesity. Eur J Hum Genet 2012;20:1290-1294.

16 Gurzov EN, Tran M, Fernandez-Rojo MA, Merry TL, Zhang X, Xu Y, Fukushima A, Waters MJ, Watt MJ, Andrikopoulos S, Neel BG, Tiganis T: Hepatic Oxidative Stress Promotes Insulin-STAT-5 Signaling and Obesity by Inactivating Protein Tyrosine Phosphatase N2. Cell Metab 2014;20:85-102.

17 Sukumar P, Viswambharan H, Imrie H, Cubbon RM, Yuldasheva N, Gage M, Galloway S, Skromna A, Kandavelu P, Santos CX, Gatenby VK, Smith J, Beech DJ, Wheatcroft SB, Channon KM, Shah AM, Kearney MT: Nox2 NADPH oxidase has a critical role in insulin resistance-related endothelial cell dysfunction. Diabetes 2013;62:2130-2134.

18 Chen X, Cui Z, Wei S, Hou J, Xie Z, Peng X, Li J, Cai T, Hang H, Yang F: Chronic high glucose-induced INS1beta cell mitochondrial dysfunction: a comparative mitochondrial proteome with SILAC. Proteomics 2013;13:3030-3039.

19 Kowluru A, Kowluru RA: Phagocyte-like NADPH oxidase [Nox2] in cellular dysfunction in models of glucolipotoxicity and diabetes. Biochem Pharmacol 2014;88:275-283.

20 Graciano MF, Santos LR, Curi R, Carpinelli AR: NAD(P)H oxidase participates in the palmitate-induced superoxide production and insulin secretion by rat pancreatic islets. J Cell Physiol 2011;226:1110-1117.

21 Greiner TU, Kesavan G, Stahlberg A, Semb H: Rac1 regulates pancreatic islet morphogenesis. BMC Dev Biol 2009;9:2.

22 Kowluru A: Small G proteins in islet beta-cell function. Endocr Rev 2010;31:52-78.

23 Mohammed AM, Kowluru A: Activation of apocynin-sensitive NADPH oxidase (Nox2) activity in INS-1 832/13 cells under glucotoxic conditions. Islets 2013;5:129-131.

24 Syed I, Kyathanahalli CN, Jayaram B, Govind S, Rhodes CJ, Kowluru RA, Kowluru A: Increased phagocytelike NADPH oxidase and ROS generation in type 2 diabetic ZDF rat and human islets: role of Rac1-JNK1/2 signaling pathway in mitochondrial dysregulation in the diabetic islet. Diabetes 2011;60:2843-2852.

25 Yuan H, Lu Y, Huang X, He Q Man Y, Zhou Y, Wang S, Li J: Suppression of NADPH oxidase 2 substantially restores glucose-induced dysfunction of pancreatic NIT-1 cells. FEBS J 2010;277:5061-5071.

26 Hansell P: NADPH-oxidase driven oxidative stress during experimental diabetes offsets NO-mediated regulation of renal medullary sodium transport. A potential treatment modality during type 1 diabetes. Acta Physiol (Oxf) 2013;209:94.

27 Gray SP, Di Marco E, Okabe J, Szyndralewiez C, Heitz F, Montezano AC, de Haan JB, Koulis C, El-Osta A, Andrews KL, Chin-Dusting JP, Touyz RM, Wingler K, Cooper ME, Schmidt HH, Jandeleit-Dahm: NADPH oxidase 1 plays a key role in diabetes mellitus-accelerated atherosclerosis. Circulation 2013;127:18881902.

28 Zhou H, Zhang F, Chen SH, Zhang D, Wilson B, Hong JS, Gao HM: Rotenone activates phagocyte NADPH oxidase by binding to its membrane subunit gp91phox. Free Radic Biol Med 2012;52:303-313. 


\section{Cellular Physiology Cell Physiol Biochem 2015;35:2135-2148

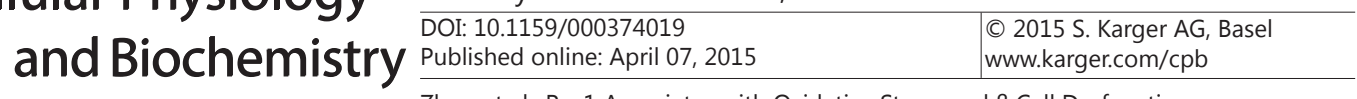 \\ Zhou et al.: Rac1 Associates with Oxidative Stress and $\beta$ Cell Dysfunction}

29 Vaquer G, Magous R, Cros G, Wojtusciszyn A, Renard E, Chevassus H, Petit P, Lajoix AD, Oiry C: Short-term intravenous insulin infusion is associated with reduced expression of NADPH oxidase p47(phox) subunit in monocytes from type 2 diabetes patients. Fundam Clin Pharmacol 2013;27:669-671.

30 Veluthakal R, Madathilparambil SV, McDonald P, Olson LK, Kowluru A: Regulatory roles for Tiam1, a guanine nucleotide exchange factor for Rac1, in glucose-stimulated insulin secretion in pancreatic betacells. Biochem Pharmacol 2009;77:101-113.

31 Tsukamoto Y, Mano T, Sakata Y, Ohtani T, Takeda Y, Tamaki S, Omori Y, Ikeya Y, Saito Y, Ishii R, Higashimori M, Kaneko M, Miwa T, Yamamoto K, Komuro I: A Novel Heart Failure Mice Model of Hypertensive Heart Disease by Angiotensin II Infusion, Nephrectomy, and Salt Loading. Am J Physiol Heart Circ Physiol 2013;305:1658-1667.

32 Lopez-Haber C, Kazanietz MG: Cucurbitacin I inhibits Rac1 activation in breast cancer cells by a reactive oxygen species-mediated mechanism and independently of Janus tyrosine kinase 2 and P-Rex1. Mol Pharmacol 2013;83:1141-1154.

33 Subasinghe W, Syed I, Kowluru A: Phagocyte-like NADPH oxidase promotes cytokine-induced mitochondrial dysfunction in pancreatic beta-cells: evidence for regulation by Rac1. Am J Physiol Regul Integr Comp Physiol 2011;300:R12-20.

34 Pi J, Collins S: Reactive oxygen species and uncoupling protein 2 in pancreatic beta-cell function. Diabetes Obes Metab 2010;12:S141-148.

35 Nozaki S, Takeda T, Kitaura T, Takenaka N, Kataoka T, Satoh T: Akt2 regulates Rac1 activity in the insulindependent signaling pathway leading to GLUT4 translocation to the plasma membrane in skeletal muscle cells. Cell Signal 2013;25:1361-1371.

36 Hwaiz R, Hasan Z, Rahman M, Zhang S, Palani K, Syk I, Jeppsson B, Thorlacius H: Rac1 signaling regulates sepsis-induced pathologic inflammation in the lung via attenuation of Mac-1 expression and CXC chemokine formation. J Surg Res 2013;183:798-807. 